\title{
Monte Carlo simulation for jet fragmentation in SUSY QCD
}

\author{
V. Berezinsky \\ INFN, Laboratori Nazionali del Gran Sasso, I-67010 Assergi (AQ), Italy \\ and Institute for Nuclear Research, Moscow, Russia \\ M. Kachelriess \\ TH Division, CERN, CH-1211 Geneva 23, Switzerland \\ and IFIC-CSIC, Departament de Física Tè̀rica, Universitat de València, Spain
}

(Received 1 September 2000; published 8 January 2001)

\begin{abstract}
We present results from a new Monte Carlo simulation for jet fragmentation in QCD and SUSY QCD for large primary energies $\sqrt{s}$ up to $10^{16} \mathrm{GeV}$. In the case of SUSY QCD the simulation takes into account not only gluons and quarks as cascading particles, but also their supersymmetric partners. A new modelindependent hadronization scheme is developed, in which the hadronization functions are found from CERN LEP data. An interesting feature of SUSY QCD is the prediction of a sizable flux of the lightest supersymmetric particles (LSPs) if $R$ parity is conserved. About $10 \%$ of the jet energy is transferred to LSPs which, owing to their harder spectra, constitute an important part of the spectra for large $x=E / E_{\text {jet }}$. Spectra of protons and secondary particles (photons and neutrinos) are also calculated. These results have implications for the decay of superheavy particles with masses up to the GUT scale, which have been suggested as a source of ultrahigh energy cosmic rays.
\end{abstract}

DOI: 10.1103/PhysRevD.63.034007

\section{INTRODUCTION}

QCD, being an essential part of the standard model, successfully describes accelerator data for production of hadrons in $e^{+} e^{-}$annihilation and deep-inelastic scattering. There are two distinctive parts in these calculations: the perturbative QCD computation of the parton cascade in jets, and the parton hadronization, in which low-virtuality partons are converted non-perturbatively into hadrons.

The QCD parton cascade is usually studied in the modified leading logarithmic approximation (MLLA), where large logarithms, $\ln \left(Q^{2}\right)$ and $\ln (1 / x)$, play a crucial role (here, $Q^{2}$ is the maximum of the perpendicular momentum $k_{\perp}$, and $x$ $=k_{\|} / k_{\|}^{\max }$ ). This approximation is characterized by remarkable features.

In the MLLA, the QCD cascade has a probabilistic interpretation provided by the absence of interference terms in the tree diagrams. The color coherence effect is taken into account in the MLLA. It suppresses the emission of soft gluons and results in the Gaussian peak of the parton distribution in terms of $\xi=\ln (1 / x)$ (hump-backed plateau).

The evolution of parton cascades in the MLLA (as well as in the LLA) is adequately described by the DokshitzerGribov-Lipatov-Altarelli-Parisi (DGLAP) equations [1].

The parton spectra can be obtained either analytically or by Monte Carlo (MC) simulations.

Examples for analytical solutions are the limiting spectrum [2] and the Gaussian spectrum [3], in which we include the distorted Gaussian spectrum $[4,5]$. The limiting spectrum is the most accurate of them. Since we have a special interest in it, we shall shortly review below the basic assumptions under which this solution is obtained.

The limiting spectrum gives the energy spectrum of partons $D_{\lim }(\xi, Y)$ for a given center-of-mass energy $\sqrt{s}$ of an $e^{+} e^{-}$-pair. Here $D$ is the number of cascade partons, $Y$ $=\ln (\sqrt{s} / 2 \Lambda)$ and $\Lambda$ is the dimensional QCD scale.

The analytical expression for $D_{\lim }(\xi, Y)$ is given in Refs. $[5,6]$. There are two fundamental parameters involved in the limiting spectrum solution: the scale $\Lambda$ and the minimal virtuality $Q_{0}$ of partons, down to which the cascade develops perturbatively; $Q_{0}$ can be viewed as the effective mass of the partons. Two assumptions are necessary for the validity of the limiting spectrum. The QCD coupling constant $\alpha_{s}\left(k_{\perp}^{2}\right)$ evolves with $k_{\perp}^{2}$ effectively, as in the one-loop approximation with three flavors $n_{f}=3$ for all $k_{\perp}^{2}$,

$$
\alpha_{s}\left(k_{\perp}^{2}\right)=\frac{12 \pi}{\left(33-2 n_{f}\right) \ln \left(k_{\perp}^{2} / \Lambda^{2}\right)} .
$$

As a matter of fact, $\Lambda$ in Eq. (1) is treated in the limiting spectrum solution as a free parameter to fit the $e^{+} e^{-}$-data. The best fit corresponds to $\Lambda=250-270 \mathrm{MeV}$. For this range of $\Lambda$ values, $\alpha_{s}\left(M_{Z}\right)$, given by Eq. (1) with $n_{f}=3$, is in the interval $0.118-0.120$, to be compared with the average experimental value $\alpha_{s}\left(M_{Z}\right)=0.1184 \pm 0.0031$ [7]. Therefore, the phenomenological parameter $\Lambda$ coincides well with $\Lambda_{\mathrm{QCD}}$, which fits the experimental value of $\alpha_{s}\left(M_{Z}\right)$ in the one-loop approximation with $n_{f}=3$.

The second assumption, necessary for the derivation of the limiting spectrum, is $Q_{0}=\Lambda$. It gives a reasonable value of $Q_{0}$, but the exact equality of these values has no theoretical justification.

The limiting spectrum solution is valid only for small $x$ $\ll 1$. In this region, which includes the maximal values of multiplicity (in the Gaussian peak), it very accurately describes experimental data at all available energies $\sqrt{s}$ (see, e.g., $[8,9]$ ). The large $x$ up to $x=1$ gives the dominant contribution to the total momentum of cascade partons. There- 
fore, the limiting spectrum solution does not guarantee that $\int x D_{\lim }(x, s) \mathrm{d} x$ precisely equals 2 .

Monte Carlo (MC) simulations of the QCD cascade give a more precise description of the cascade evolution. For example, in contrast to the limiting spectrum, in MC simulations one can use $\alpha_{s}\left(k_{\perp}^{2}\right)$ with the measured value of $\Lambda_{\mathrm{QCD}}$, varying the number of flavors and two-loop corrections being taken into account. The assumption $\Lambda=Q_{0}$, specific to the limiting spectrum, is not needed. MC simulations are based on a probabilistic interpretation of the jet cascade. Parton branching is described by the Altarelli-Parisi functions, and the probability of parton evolution between two values of virtualities without branching is given by the Sudakov form factor. Finally, the coherent effect in the soft gluon emission (destructive interference) is conveniently taken into account by angular ordering $\theta_{1}>\theta_{2}>\theta_{3} \ldots[10]$, where the indices number the generations (non-ordered processes are suppressed [11]). The first MC simulation with angular ordering was performed in Ref. [10]. At present there are several detailed MC simulations, e.g. [12-15], which differ mainly in their description of hadronization. We shall now briefly discuss the problem of hadronization.

The description of parton hadronization is based on the assumption of local parton-hadron duality (LPHD) [16]. This hypothesis implies that when $Q_{0}$ is small enough (of the order of $\Lambda$ ) there is a proportionality between the spectra of partons and hadrons, with relations between their momenta, which are local in the phase space. Such an interpretation can be justified by the idea of preconfinement [17].

As far as spectra are concerned, LPHD implies a proportionality between the hadron and parton spectra. In Refs. $[5,6]$, it is emphasized that, most reasonably, this proportionality holds not on a one parton-one hadron basis, but for the number of particles averaged over a finite interval $\Delta \xi \sim 1$.

The LPHD hypothesis for limiting spectrum results straightforwardly [5] in

$$
D_{\text {had }}(x, \sqrt{s})=K_{h}\left(Q_{0}\right) D_{\text {part }}\left(x, \sqrt{s}, Q_{0}\right),
$$

where the constant $K_{h}$ is universal, in the sense that it does not depend on $\sqrt{s}$.

Equation (2) completes our description of the limiting spectrum, expressing the hadron spectrum through the spectrum of partons. The constant $K_{h}$, which connects the two spectra, is found from a comparison with experimental data as $K_{h} \approx 1.3$ for $\Lambda=Q_{0} \approx 270 \mathrm{MeV}$ [8], and it does not change with energy unless some new physics (e.g. supersymmetry) appears.

In MC simulations, the parameter $Q_{0}$ is, in principle, a free parameter found by fitting experimental data. For HERWIG [12] and PYTHIA [13], for example, $Q_{0} \sim 1 \mathrm{GeV}$. Several detailed hadronization models are used in simulations, e.g. the independent fragmentation model, the Lund string model [18], and the cluster fragmentation model [19]. Usually, these models use many free parameters and require us to keep track of the four-momentum evolution of all partons.

The calculations described above are valid up to $\sqrt{s}$ $\sim 1-10 \mathrm{TeV}$. At higher energies the production of supersymmetric particles is expected to change the results. One might be interested in much higher energies, being inspired by the production of superheavy particles up to the grand unified theory (GUT) scale in the Universe. Such particles can be produced by both topological defects and many processes at the post-inflationary stage of the Universe. Recently, superheavy particles with masses $M_{X} \sim 10^{12}-10^{14}$ $\mathrm{GeV}$ attracted much attention as a source of the observed ultrahigh energy cosmic rays (UHECR) with energies $10^{19}-10^{20} \mathrm{eV}$ (for recent reviews, see [20]).

The limiting spectrum for SUSY QCD was calculated in Ref. [21] for very high energies $\sqrt{s}$, corresponding to masses of superheavy particles $M_{X} \sim 10^{12}-10^{14} \mathrm{GeV}$. The supersymmetric partons (squarks and gluinos or jointly spartons) participate in the cascade until the virtualities $t$ of the particles drop below the mass scale of SUSY particles, $t \sim M_{\text {SUSY }}^{2}$. Then a SUSY particle decays, producing in the end the lightest supersymmetric particle (LSP), for which the lightest neutralino is usually considered. The role of supersymmetric partners is twofold: they double the number of parton types in the cascade, and they change the evolution of $\alpha_{s}\left(k_{\perp}^{2}\right)$. Even at small $t \ll M_{\text {SUSY }}^{2}$, the cascade remembers the number of flavors at large $t$ because, for example, each squark leaves after its decay a quark, which continues QCD cascading. At large $t$ and small $x \ll 1$, gluons and gluinos dominate and their "children" constitute the dominant part of the cascade at small $t$. Therefore, the dominant contribution to the limiting spectrum is given by gluons and gluinos.

The SUSY QCD limiting spectrum solution has two drawbacks with respect to ordinary QCD. First, the number of flavors that determine the evolution of the coupling constant according to Eq. (1) has to be fixed to one value of $n_{f}$ for the whole range of $k_{\perp}^{2}$. Second, the limiting spectrum for ordinary QCD is normalized by experimental data, which are absent in the case of SUSY QCD. Normalization due to the conservation of momentum $\int x D_{\lim }(x, s) \mathrm{d} x=2$ is unreliable since the limiting spectrum is not valid for large $x$, which gives the main contribution to the integral (see the discussion in [21]).

During the last few years, the production and decays of supersymmetric particles have been included in most MC simulations focusing on the CERN Large Hadron Callider (LHC) studies. Although the LHC will operate above the expected threshold of SUSY particle production, its energy is not large enough for these particles to participate in the QCD cascade. Therefore, all currently available MC simulations consider only on-shell decays of spartons and neglect possible branchings of gluinos and squarks. ${ }^{1}$ Another obstacle against the use of standard MC simulations at extremely large energies around $\sqrt{s} \sim 10^{12}-10^{14} \mathrm{GeV}$ is that the necessary numerical precision and required amount of memory space and computing time become a challenge for presentday computers.

We have therefore developed a new MC simulation, which includes as cascading particles not only gluons and

\footnotetext{
${ }^{1}$ The future $\mathrm{C}++$ version of HERWIG will include branchings of spartons [23]
} 
quarks, but also gluinos and squarks. We consider cascades that are initiated by the decay to two jets of superheavy particles with mass $M_{X} \sim 10^{12}-10^{14} \mathrm{GeV}$, or by $e^{+} e^{-}$annihilation at $s=M_{X}^{2}$. SUSY partons, squarks and gluinos are produced in the fragmentation of ordinary partons, and vice versa. All squarks and gluinos are assumed to have equal masses, for which we use $M_{\text {SUSY }}=200 \mathrm{GeV}$ and $M_{\text {SUSY }}$ $=1000 \mathrm{GeV}$. When the virtuality of the cascading particles drops below $M_{\text {SUSY }}^{2}$, sparticles decay to LSPs (neutralinos), which freely escape. The perturbative development of the cascade continues with ordinary partons until their virtualities reach $Q_{0}^{2}$, for which we use $Q_{0}^{2}=0.625 \mathrm{GeV}^{2}$ to fit the data at small energies $\sqrt{s}$. We use a new hadronization procedure. It is based on a model-independent, phenomenological approach, in which hadronization functions for large $s$ (or $M_{X}$ ) are calculated from hadron spectra observed at small $s$ $\left(M_{X}\right)$. This method can be used for any type of hadron, as well as for photons and neutrinos, if their spectra are known with good enough accuracy at small energy.

Following Ref. [22], we shall use the following notation:

$\Lambda$ is the dimensional QCD scale,

$Y=\ln (\sqrt{s} / 2 \Lambda)$,

$t=p_{\mu}^{2}$ is the virtuality of cascade partons,

$Q^{2}=t_{\max }$ is the virtuality of the primary parton,

$Q_{0}^{2}$ is the minimum virtuality of the perturbative evolution of the QCD cascade,

$z=E^{\prime} / E$, where $E$ and $E^{\prime}$ are the energies of ingoing and outgoing partons at fragmentation,

$\zeta=1-\cos \theta$, where $\theta$ is the angle between two outgoing partons,

$\tilde{t}=\zeta E^{2}$,

$k_{\perp}, k_{\|}$are the transverse and parallel momenta transferred, respectively,

$$
x=k_{\|} / k_{\|}^{\max },
$$$$
\xi=\ln (1 / x) \text {. }
$$

\section{MC SIMULATION OF THE PERTURBATIVE PHASE OF SUSY QCD CASCADES}

The perturbative part of our simulation is very similar to those of MC simulations for ordinary QCD cascades, except for including spartons and the condition for their exit from the cascade. We consider a superheavy $X$ particle with mass $M_{X}$, which decays into two jets with energy $E_{\text {jet }}=M_{X} / 2$. We assume that the primary partons produced in the $X$ particle decay have the maximum virtuality $Q^{2}=m_{X}^{2} / 4$ and that the $X$ particle has equal branching ratios to all partons. As to the first assumption, in reality, there is a distribution of partons with different $t$, but the Sudakov form factors suppress small $t$ values. The second assumption is made because of the unspecified interactions of the $X$ particles.

Our simulation closely follows the angular ordered parton shower algorithm developed in Refs. $[10,19]$. In this algorithm it is convenient to use the variable $\tilde{t}=\zeta E^{2}$, where $E$ is the energy of the incoming parton, $\zeta \approx 1-\cos \theta$, and $\theta$ is the angle between the two emitted partons. A primary parton with energy $E_{\text {jet }}\left(=m_{X} / 2\right)$ and angular variable $\xi_{0} \leqslant 1$ initiates a cascade, which proceeds until the ordinary partons
TABLE I. Splitting functions $P_{i \rightarrow j k}(z)$, where $z$ is the energy fraction of the particle $j$.

\begin{tabular}{cc}
\hline \hline splitting channel $i \rightarrow j k$ & splitting function $P_{i \rightarrow j k}(z)$ \\
\hline$g \rightarrow g+g$ & $3\left[\frac{z}{1-z}+\frac{1-z}{z}+z(1-z)\right]$ \\
$g \rightarrow \tilde{g}+\tilde{g}$ & $3\left[z^{2}+(1-z)^{2}\right]$ \\
$g \rightarrow q+q$ & $\frac{n_{f}^{*}}{2}\left[z^{2}+(1-z)^{2}\right]$ \\
$g \rightarrow \tilde{q}+\tilde{q}$ & $3\left\{1-\left[z^{2}+(1-z)^{2}\right]\right\}$ \\
$\tilde{g} \rightarrow g+\tilde{g}$ & $3 \frac{1+(1-z)^{2}}{z}$ \\
$\tilde{g} \rightarrow \tilde{q}+q$ & $3 z$ \\
$q \rightarrow q+g$ & $\frac{4}{3} \frac{1+z^{2}}{1-z}$ \\
$q \rightarrow \tilde{q}+\tilde{g}$ & $\frac{4}{3} z$ \\
$\tilde{q} \rightarrow \tilde{q}+g$ & $\frac{4}{3}\left[\frac{1+z^{2}}{1-z}-(1-z)\right]$ \\
$\tilde{q} \rightarrow q+\tilde{g}$ & $\frac{4}{3}$ \\
\hline \hline
\end{tabular}

reach the minimal virtuality $\tilde{t}=4 Q_{0}^{2}$. Here the perturbative evolution of the cascade terminates.

In each branching of an incoming parton $i$ with $\tilde{t}^{\prime}$, we generate with the veto algorithm [24], a new $\tilde{t}$ and $z$ according to the probability distribution

$$
\mathrm{d} \mathcal{P}_{i}(\tilde{t}, z)=\sum_{j k} \frac{\mathrm{d} \tilde{t}}{\tilde{t}} \frac{\mathrm{d} z}{2 \pi} \alpha_{s}\left[z^{2}(1-z)^{2} \tilde{t}\right] P_{i \rightarrow j k}(z) \frac{\Delta_{i}\left(\tilde{t}^{\prime}\right)}{\Delta_{i}(\tilde{t})} .
$$

Here, the sum includes all possible branching channels $j k$, $z^{2}(1-z)^{2} \tilde{t}$ is the parton transverse momentum, and $\Delta_{i}$ is the product of the individual Sudakov-like form factors $\Delta_{i \rightarrow j k}$ [19],

$$
\Delta_{i \rightarrow j k}(\tilde{t})=\exp \left[-\int_{4 \tilde{t}_{\min }}^{\tilde{t}} \frac{\mathrm{d} t^{\prime}}{t^{\prime}} f_{i \rightarrow j k}\left(t^{\prime}\right)\right]
$$

with

$$
f_{i \rightarrow j k}(\tilde{t})=\int_{z_{\min }}^{z_{\max }} \frac{\mathrm{d} z}{2 \pi} \alpha_{s}\left[z^{2}(1-z)^{2} \tilde{t}\right] P_{i \rightarrow j k}(z) .
$$

The unregularized Altarelli-Parisi splitting functions $P_{i \rightarrow j k}(z)$ of SUSY QCD [25] are given in Table I.

The angular ordering $\zeta_{j}, \zeta_{k}<\zeta_{i}$ for the branching $i \rightarrow j k$, which takes into account color coherence, is equivalent to $\tilde{t}_{j}<z^{2} \widetilde{t}_{i}$ and $\tilde{t}_{k}<(1-z)^{2} \widetilde{t}_{i}$. These conditions result in

$$
z_{\min }=\sqrt{\tilde{t}_{\min } / \tilde{t}}, \quad z_{\max }=1-\sqrt{\tilde{t}_{\min } / \tilde{t}} .
$$


For the evolution of the running coupling $\alpha_{s}$ as a function of gluon virtuality $t$ at small momentum transfer $t<t_{\text {SUSY }}$, we use the standard two-loop dependence with variable $n_{f}$ and thresholds, and normalize $\alpha_{s}$ as $\alpha_{s}\left(M_{Z}\right)=0.119$, which corresponds to $\Lambda_{\overline{\mathrm{MS}}}^{(5)}=222 \mathrm{MeV}$. At large momentum transfer $t$ $>t_{\text {SUSY }}$ we use a minimal SUSY-SU(5) coupling constant evolution [26], normalizing the coupling constant at $\sqrt{t}$ $=M_{\mathrm{GUT}}=1 \times 10^{16} \mathrm{GeV}$, as $\alpha_{s}\left(M_{\mathrm{GUT}}^{2}\right) \approx 1 / 25.8$. Explicitly we use

$$
\alpha_{s}(t)=\frac{\alpha\left(M_{\mathrm{GUT}}^{2}\right)}{1+b_{s} /(4 \pi) \ln \left(t / M_{\mathrm{GUT}}^{2}\right) \alpha\left(M_{\mathrm{GUT}}^{2}\right)},
$$

where $b_{s}=9-n_{f}$ is a constant that governs the evolution of the coupling constant with $t$. At $t>t_{\mathrm{SUSY}}, n_{f}=6$ and $b_{s}=3$. The above assumption means that we introduce, instead of many thresholds corresponding to SUSY particles with different masses, a single threshold at $t=t_{\mathrm{SUSY}}$. This is a reasonable thing to do in view of the large uncertainties in our knowledge of mass spectrum of SUSY particles. Equation (7) approximates accurately enough the evolution of $\alpha_{s}(t)$ as calculated in Ref. [27], when $t_{\mathrm{SUSY}} \approx 2 \times 10^{5} \mathrm{GeV}^{2}$. Starting from this value, $\alpha_{s}(t)$ evolves in the regime of Eq. (7). Note that $t_{\text {SUSY }}$ does not necessarily coincide with the scale $M_{\text {SUSY }}$, the universal mass of squarks and gluinos, for which we use as two representative values $M_{\text {SUSY }}=200 \mathrm{GeV}$ and $M_{\text {SUSY }}=1 \mathrm{TeV}$. In particular, the low value of $t_{\mathrm{SUSY}}$ used here is compatible with much larger $M_{\text {SUSY }}$, as emphasized in Ref. [27].

Finally, we have to specify the value of the cutoff $\tilde{t}_{\min }$ for the shower evolution. We do not distinguish between different quark flavors, and we use $\tilde{t}_{\text {min }}=0.625 \mathrm{GeV}^{2}$ for all branchings in which only normal particles are produced, and $\tilde{t}_{\text {min }}=M_{\text {SUSY }}^{2}$, where $M_{\text {SUSY }}$ is the typical mass scale of the spartons, for branchings in which SUSY particles are produced, respectively.

Let us now describe a step $i \rightarrow j k$ in our simulation. For an incoming parton $i$ with $\tilde{t}^{\prime}$, we first generate a new cascade variable $\widetilde{t}$, according to the probability distribution given by the ratio $\Delta_{i}\left(\tilde{t}^{\prime}\right) / \Delta_{i}(\widetilde{t})$. Then we select the branching channel $j k$ using $f_{i \rightarrow j k}(\tilde{t})$ as weight, and generate $z$ according to the probability distribution $\alpha_{s}\left[z^{2}(1-z)^{2} \tilde{t}\right] P_{i \rightarrow j k}(z)$.

The last ingredient in the perturbative part of our simulation is the exit of supersymmetric particles from the cascade. We assume that the neutralino $\tilde{\chi}$ is the LSP and that $R$-parity is conserved. Reaching $\tilde{t}_{\text {min }}=M_{\text {SUSY }}^{2}$, squarks and gluinos decay as $\tilde{q} \rightarrow q+\tilde{\chi}$ and $\tilde{g} \rightarrow q+\bar{q}+\tilde{\chi}$, thus producing UHE LSPs.

In this work we are running two Monte Carlo similations: one with ordinary QCD and one with SUSY QCD.

In the former case supersymmetric partons are not included and, for perturbative calculations, we assume the SM particle content with $\alpha_{s}(t)$ evolution in two-loop approximation with proper thresholds. We fix $Q_{0}^{2}=0.625 \mathrm{GeV}^{2}$. We need these calculations mostly for the test of our method.
The assumptions of SUSY QCD Monte Carlo simulations are described above. At $\tilde{t}_{\min }<M_{\mathrm{SUSY}}^{2}$ cascade develops according to the ordinary QCD scheme.

\section{HADRONIZATION}

The Monte Carlo simulation described in the last section is completely determined by perturbative physics. How the spectrum of colored quarks and gluons $D_{i}(x, \sqrt{s})$ is transformed into the spectrum of hadrons $D_{\text {had }}(x, \sqrt{s})$ is still an open problem. Monte Carlo simulations have to use some hadronization model (see Introduction), which describes the non-perturbative evolution of the cascade for $\tilde{t}<4 \tilde{t}_{\min }$. Two hadronization models, the cluster fragmentation model [19] used in HERWIG [12] and the Lund string model [18] used in PYTHIA [13], require the knowledge of the four-momenta of all the partons. Thus these models need detailed time and memory-consuming computations.

We suggest here a phenomenological, model-independent hadronization scheme based on the knowledge of the hadron spectra at energies $\sqrt{s}$ smaller than the energy of interest. This method is valid for any hadron type and can be applied to the secondary particles, such as photons and neutrinos, as well. The application of this method is somewhat restricted (e.g. it cannot give the angular distribution of particles in a jet or correlations), but its use is very efficient for the decay of superheavy particles, where multiplicity, and hence the number of partons to follow in a simulation, is very large.

Our hadronization scheme depends on only one theoretical assumption, which is reliable and testable. Namely, we assume that the unknown non-perturbative physics can be factorized into hadronization functions $f_{i}(z)$ that do not depend on $\sqrt{s}$,

$$
D_{h}(x, \sqrt{s})=\sum_{i=q, g} \int_{x}^{1} \frac{\mathrm{d} z}{z} D_{i}(x / z, \sqrt{s}) f_{i}^{h}(z)
$$

where the index $h$ runs through different types of hadrons, e.g. $\pi^{0}, \pi^{ \pm}, N$, etc.

The functions $f_{i}^{h}(z)$ give the probability that a parton $i$ with energy $E$ is converted into a hadron $h$ with energy $z E$. It is implicitly assumed in Eq. (8) that the perturbative cutoff $Q_{0}$ is fixed, and $f_{i}^{h}$ is determined for this value of $Q_{0}$, although in principle, for every $Q_{0}$ and $D_{i}\left(x, \sqrt{s}, Q_{0}\right)$ one can find $f_{i}^{h}\left(z, \sqrt{s}, Q_{0}\right)$ to fit the observed hadron spectra.

Equation (8), with energy independent hadronization functions, follows from basic principles and is confirmed (see below) at energies of $e^{+} e^{-}$colliders. It has the form of a Volterra integral equation of the first kind though, in contrast to the standard case, the right-hand side contains not one but two unknown functions $f_{i}$ for every $h$. In principle, the two functions $f_{g}(x)$ and $f_{q}(x)$ can be uniquely determined if $D_{h}$ is known as an analytic function without errors for two different values of $\sqrt{s}$. In practice, $D_{h}(x)$ is known only as a discrete set of experimental data and Eq. (8) rep- 


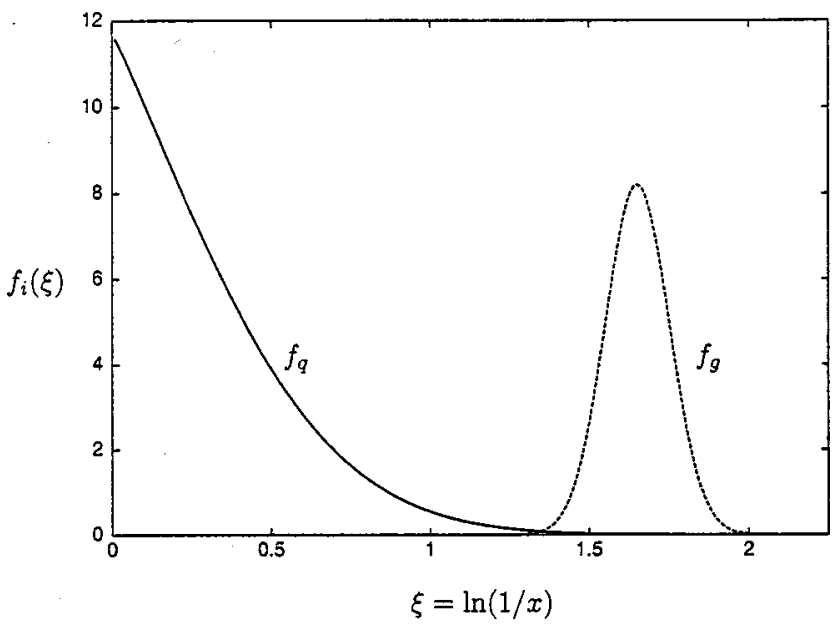

FIG. 1. Hadronization functions for quarks $f_{q}(\xi)$ (solid line) and gluons $f_{g}(\xi)$ (broken line) obtained by fitting Gaussians to experimental data at $\sqrt{s}=91.2 \mathrm{GeV}$.

resents an ill-posed inversion problem ${ }^{2}$ [28]. Instead of solving Eq. (8) by an inversion method, we prefer to find physically motivated trial functions for $f_{i}$ to fit the experimental data at $\sqrt{s}=91.2 \mathrm{GeV}$.

In terms of the more convenient variable $\xi=\ln (1 / x)$, Eq. (8) has the form

$$
D_{h}(\xi, \sqrt{s})=\sum_{i=q, g} \int_{0}^{l} \mathrm{~d} \xi^{\prime} D_{i}\left(\xi-\xi^{\prime}, \sqrt{s}\right) f_{i}\left(\xi^{\prime}\right)
$$

where the index $h$ in the hadronization functions is suppressed.

In the limiting spectrum, when $Q_{0}=\Lambda$, the hadronization functions $f_{i}$ are proportional to delta functions. Inspired by this analytical solution, we choose for $f_{i}$ Gaussian functions

$$
f_{i}(\xi)=a_{i} \exp \left(-\frac{\left(\xi-\xi_{\max , i}\right)^{2}}{\sigma_{i}^{2}}\right) .
$$

With this hadronization function the approximate proportionality holds between spectra of partons and hadrons as LPHD demands. The position of the peak in the hadronization function determines the shift between the maxima of parton and hadron spectra. While for gluons the hadronization function $f_{g}(\xi)$ should vanish for $\xi \rightarrow 0$, because gluons have to split their energy to a $q \bar{q}$ pair, for quarks, $f_{q}(\xi)$ can be finite at $\xi=0$.

The hadronization functions we obtained for $Q_{0}^{2}=0.625$ $\mathrm{GeV}^{2}$ from a fit to LEP data at $\sqrt{s}=91.2 \mathrm{GeV}$ are shown in Fig. 1.

\footnotetext{
${ }^{2}$ Volterra integral equations of the first kind can be solved normally by linearization, even if the left-hand side is data. However, the lower integration limit in Eq. (8) does not represent a sharp cutoff because the kernels $D_{i}(x)$ vanish for $x \rightarrow 1$. Therefore, Eq. (8) behaves effectively like a Fredholm equation, and these are known to be extremely ill-conditioned.
}

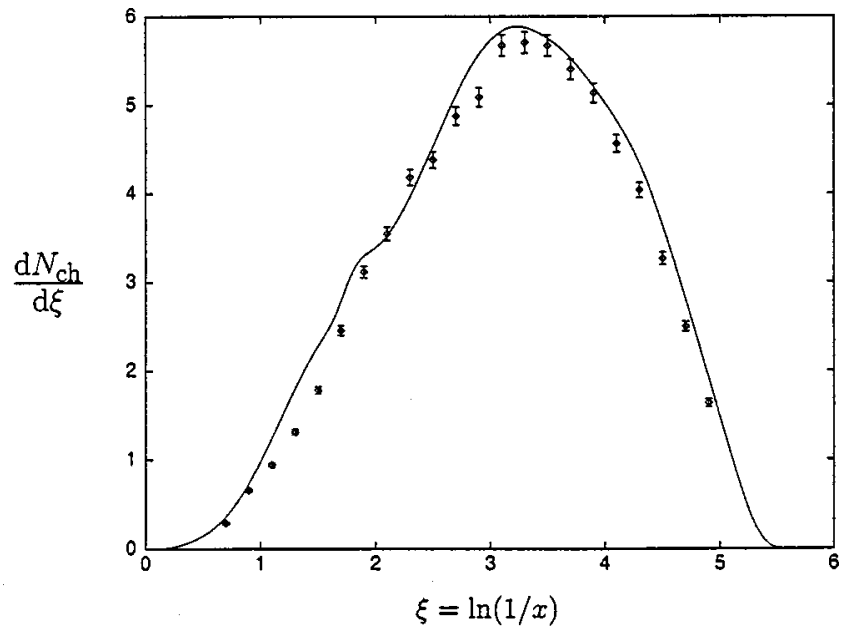

FIG. 2. Comparison of the spectrum of charged hadrons $\mathrm{d} N_{\mathrm{ch}} / \mathrm{d} \xi$ from an ordinary QCD Monte Carlo simulation (solid line) with the experimental data (shown with error bars) at $\sqrt{s}=58 \mathrm{GeV}$.

Our hadronization scheme has been tested by two methods: for relatively small energies, $\sqrt{s}=58 \mathrm{GeV}$ and 133 $\mathrm{GeV}$, we confronted our calculations with the CERN $e^{+} e^{-}$ collider LEP data, and for very large $\sqrt{s}$ (or $M_{X}$ ), we compared the calculated spectrum with the limiting spectrum using a special case when it is correct (see below). In both cases ordinary QCD Monte Carlo simulations were used.

Figures 2-4 display a comparison between the charged hadron spectrum from our MC simulation for ordinary QCD and experimental data [29] at $\sqrt{s}=58,91.2$ and $133 \mathrm{GeV}$, respectively.

Let us now discuss whether the hadronization functions $f_{i}(\xi)$ found from the fit to data at $\sqrt{s}=91.2 \mathrm{GeV}$ can really be used at $M_{X}=10^{12}-10^{16} \mathrm{GeV}$.

First of all, we note that a test can be given by LPHD, which demands approximate proportionality between parton and hadron spectra. It implies that the $\xi^{\prime}$ values that give the

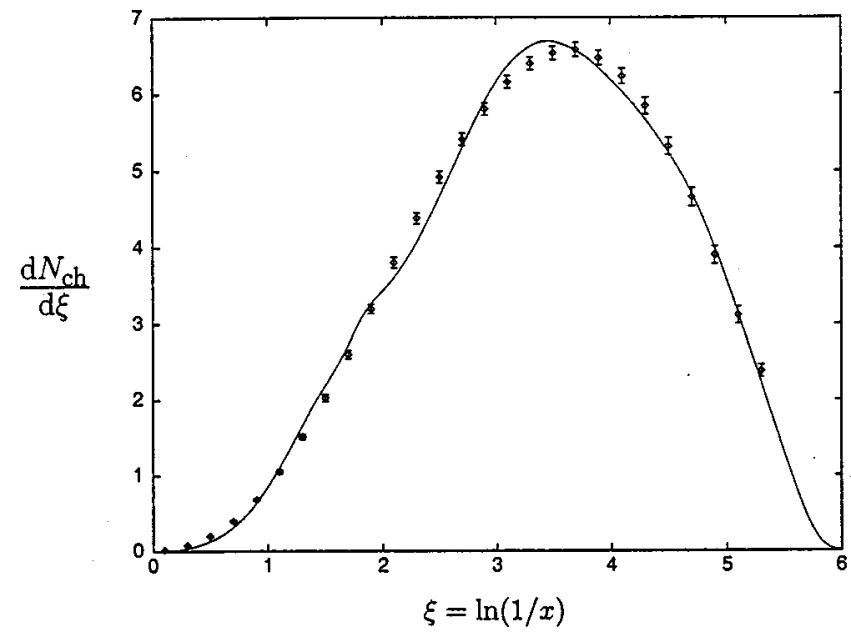

FIG. 3. Comparison of the spectrum of charged hadrons $\mathrm{d} N_{\mathrm{ch}} / \mathrm{d} \xi$ from an ordinary QCD Monte Carlo simulation (solid line) with the experimental data (shown with error bars) at $\sqrt{s}=91.2$ $\mathrm{GeV}$. 


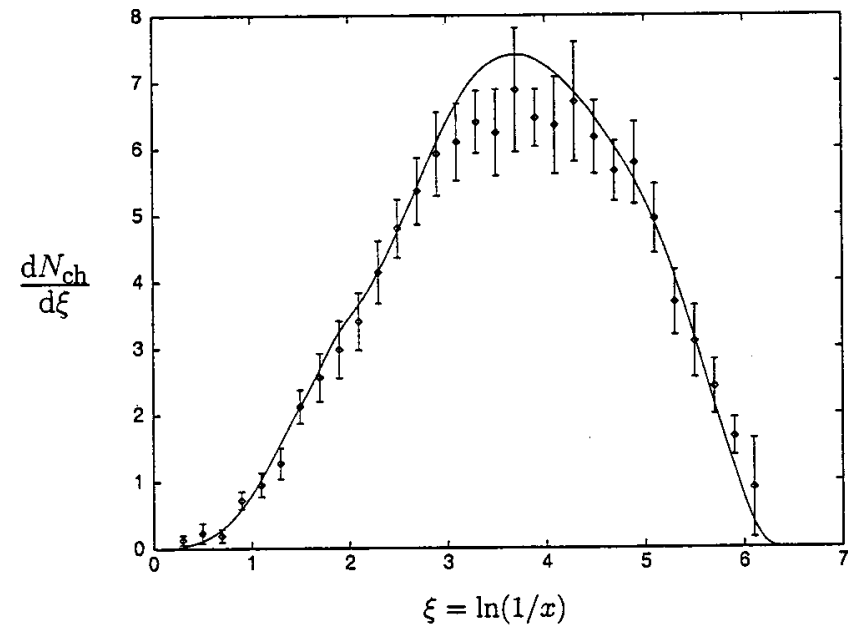

FIG. 4. Comparison of the spectrum of charged hadrons $\mathrm{d} N_{\mathrm{ch}} / \mathrm{d} \xi$ from an ordinary QCD Monte Carlo simulation (solid line) with the experimental data (shown with error bars) at $\sqrt{s}=133$ $\mathrm{GeV}$.

dominant contribution to the integral in Eq. (9) are about the same at $\sqrt{s}=91.2 \mathrm{GeV}$ and at large $M_{X}$. Numerical tests show that this is indeed the case for both the quark and the gluon contribution.

As a critical test of our hadronization scheme, we compared the results of our simulation with the limiting spectrum in a special, well-controlled case of ordinary QCD with the number of quark flavors $n_{f}=3$, and with $\alpha_{s}\left(k_{\perp}^{2}\right)$ given by Eq. (1). For the limiting spectrum in this case, we can use the normalization constant $K_{h} \approx 1.3$ obtained by fitting experimental data [9]. In Fig. 5, we show the ratio of these two (charged) hadron spectra. The agreement between the two spectra is excellent, except for the small $\xi \lesssim 6$ region where it is known that the limiting spectrum is not valid. The disagreement reaches $50 \%$ at $\xi \approx 2.1(x \approx 0.12)$.

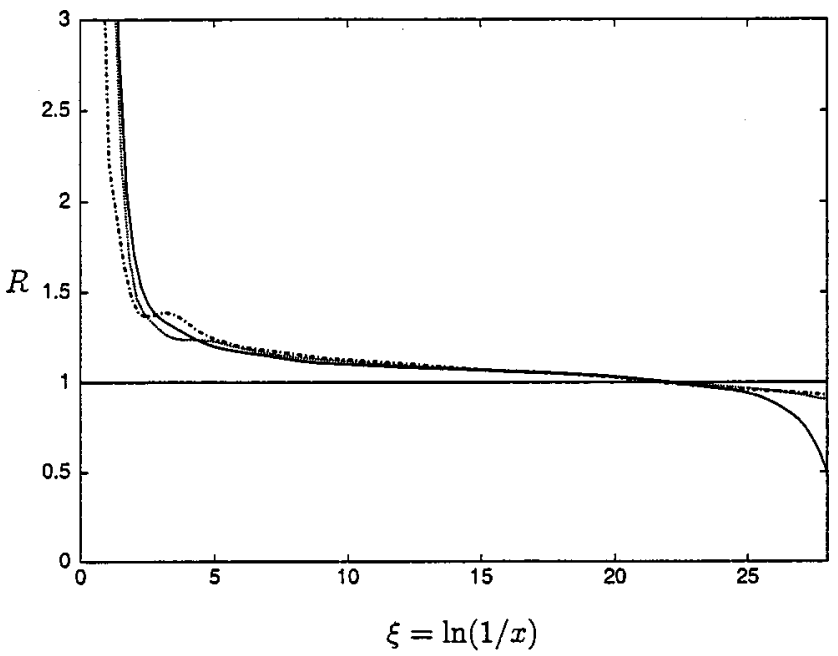

FIG. 5. The ratio $R=D_{\lim }(\xi) / D_{\mathrm{MC}}(\xi)$ of the limiting spectrum and the hadron spectrum from the simulation for $M_{X}=10^{12} \mathrm{GeV}$ (solid line), $10^{13} \mathrm{GeV}$ (broken line) and $10^{14} \mathrm{GeV}$ (dashed line). All are for ordinary QCD with $n_{f}=3$.

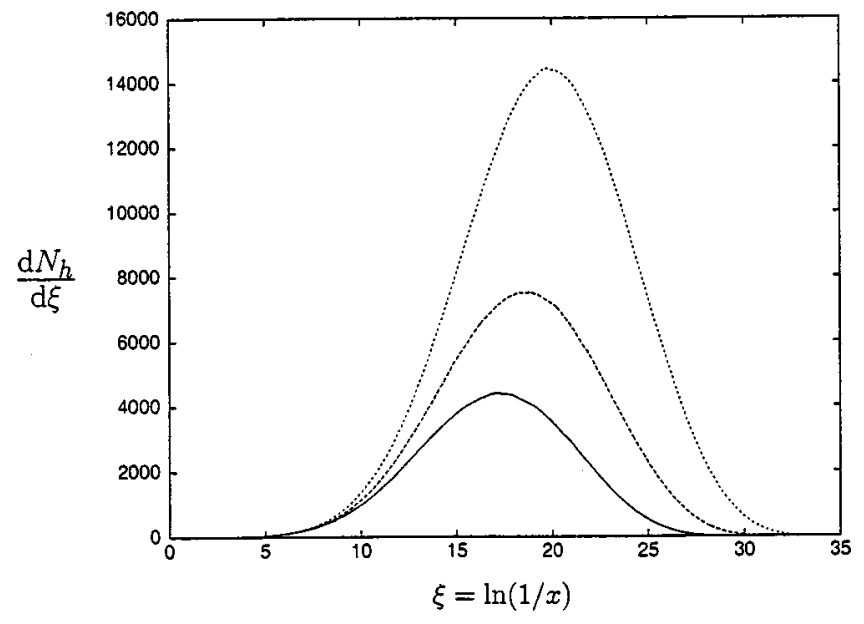

FIG. 6. Hadron spectra $\mathrm{d} N_{h} / \mathrm{d} \xi$ from the SUSY QCD Monte Carlo simulation for $M_{X}=10^{12} \mathrm{GeV}$ (bottom), $M_{X}=10^{13} \mathrm{GeV}$ (middle) and $M_{X}=10^{14} \mathrm{GeV}$ (top), with all for $M_{\text {SUSY }}=200 \mathrm{GeV}$.

In conclusion, we think that our hadronization recipe is a valid alternative to the extrapolation of the Lund string or the cluster fragmentation model to extremely large $M_{X}$.

\section{RESULTS: SPECTRA OF HADRONS AND SECONDARY PARTICLES}

Using the algorithm for the perturbative evolution of the SUSY QCD cascade as described in Sec. II, and our hadronization scheme from Sec. III, we can now compute the fragmentation spectra of hadrons. As numerical values for $M_{X}$, we choose in the graphs given as examples three values interesting for UHECR physics, $M_{X}=10^{12}, 10^{13}, 10^{14} \mathrm{GeV}$, as well as $M_{X}=10^{6}$ and $10^{16} \mathrm{GeV}$ as the lowest and highest scale of interest. Similarly, we use $M_{\text {SUSY }}=200 \mathrm{GeV}$ and $M_{\text {SUSY }}=1000 \mathrm{GeV}$ as two representative values for the SUSY mass scale.

In Figs. 6 and 7, the hadron spectra $\mathrm{d} N_{\text {had }} / \mathrm{d} \xi$ from SUSY QCD MC simulations are displayed as functions of $\xi$ for

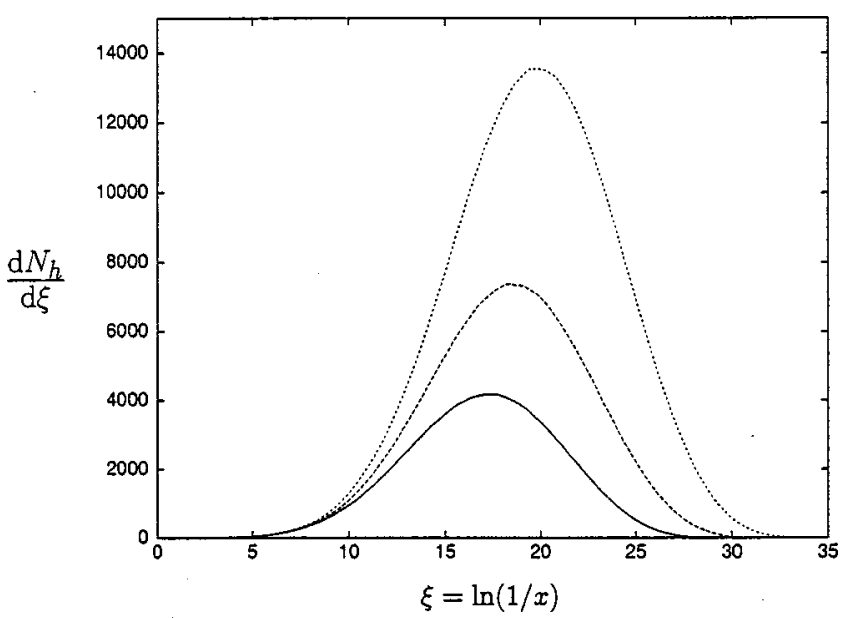

FIG. 7. Hadron spectra $\mathrm{d} N_{h} / \mathrm{d} \xi$ from the SUSY QCD Monte Carlo simulation for $M_{X}=10^{12} \mathrm{GeV}$ (bottom), $M_{X}=10^{13} \mathrm{GeV}$ (middle) and $M_{X}=10^{14} \mathrm{GeV}$ (top), with all for $M_{\mathrm{SUSY}}=1 \mathrm{TeV}$. 


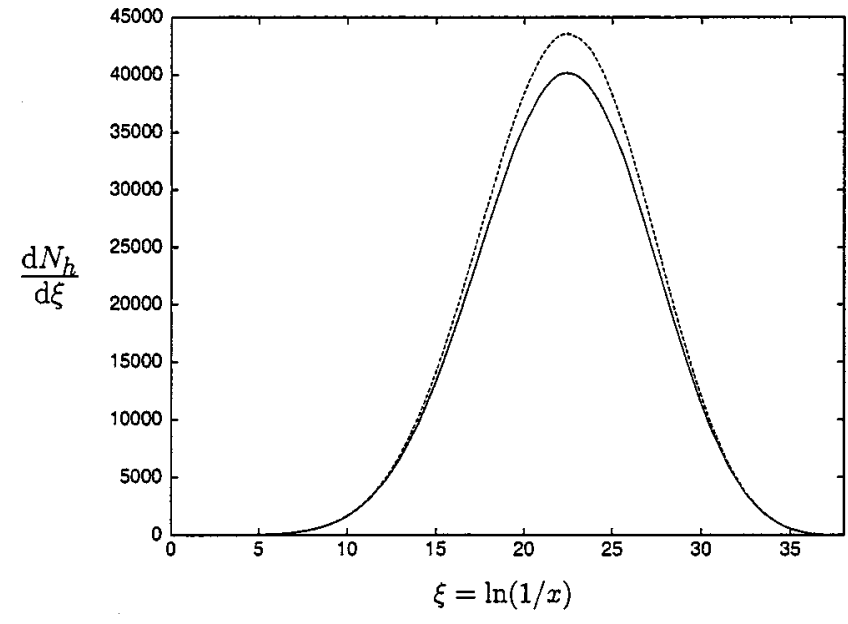

FIG. 8. Hadron spectra $\mathrm{d} N_{h} / \mathrm{d} \xi$ for the SUSY QCD Monte Carlo simulation for $M_{\text {SUSY }}=200 \mathrm{GeV}$ (broken line) and $M_{\text {SUSY }}$ $=1 \mathrm{TeV}$ (solid line), both for $M_{X}=10^{16} \mathrm{GeV}$.

$M_{\text {SUSY }}=200 \mathrm{GeV}$ and $M_{\text {SUSY }}=1000 \mathrm{GeV}$, respectively; in both figures the spectra were calculated for $M_{X}$ $=10^{12}, 10^{13}, 10^{14} \mathrm{GeV}$. For the GUT scale $M_{X}=10^{16} \mathrm{GeV}$, the hadron spectra $\mathrm{d} N_{\text {had }} / \mathrm{d} \xi$ are shown in Fig. 8, and for the low scale $M_{X}=10^{6} \mathrm{GeV}$ in Fig. 9. The hadron spectra depend only weakly on $M_{\text {SUSY }}$, with increasing differences for larger values of $M_{X}$. Both effects are easy to understand: when spartons disappear from the cascade at $\tilde{t} \sim M_{\text {SUSY }}^{2}$ due to on-shell decays, each of them leaves there an ordinary parton with similar virtuality. Therefore, the cascade proceeds as if nothing had happened, except that some energy is lost through the emission of neutralinos and leptons, which is not large $(\sim 10 \%)$. Second, the importance of spartons for the cascade decreases with smaller values of $M_{X}$, thereby also reducing the dependence of the hadron spectra on $M_{\text {SUSY }}$ for smaller $M_{X}$.

The signature of supersymmetry in decays of superheavy $X$ particles is the production of LSPs, which we assume as

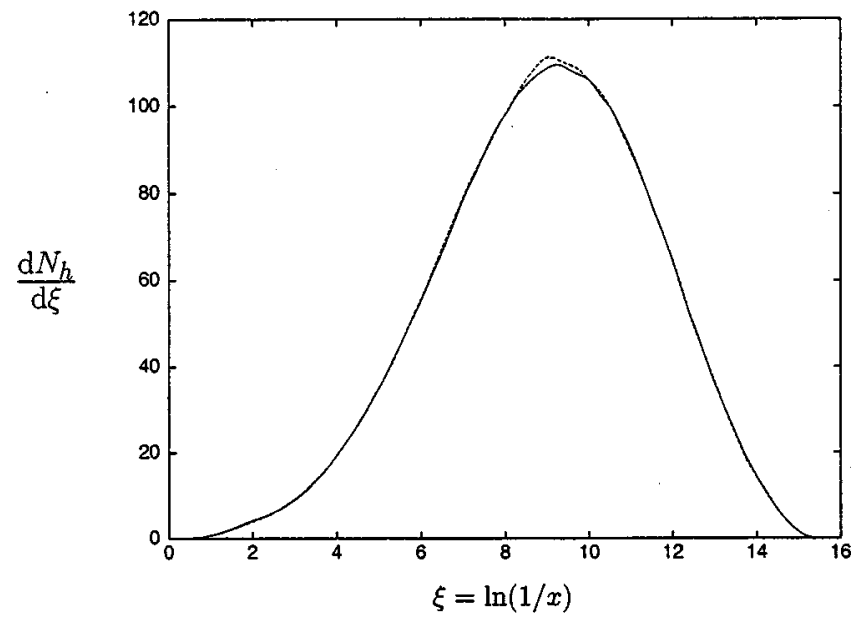

FIG. 9. Hadron spectra $\mathrm{d} N_{h} / \mathrm{d} \xi$ from the SUSY QCD Monte Carlo simulation for $M_{\mathrm{SUSY}}=200 \mathrm{GeV}$ (broken line) and $M_{\mathrm{SUSY}}$ $=1 \mathrm{TeV}$ (solid line), both for $M_{X}=10^{6} \mathrm{GeV}$.

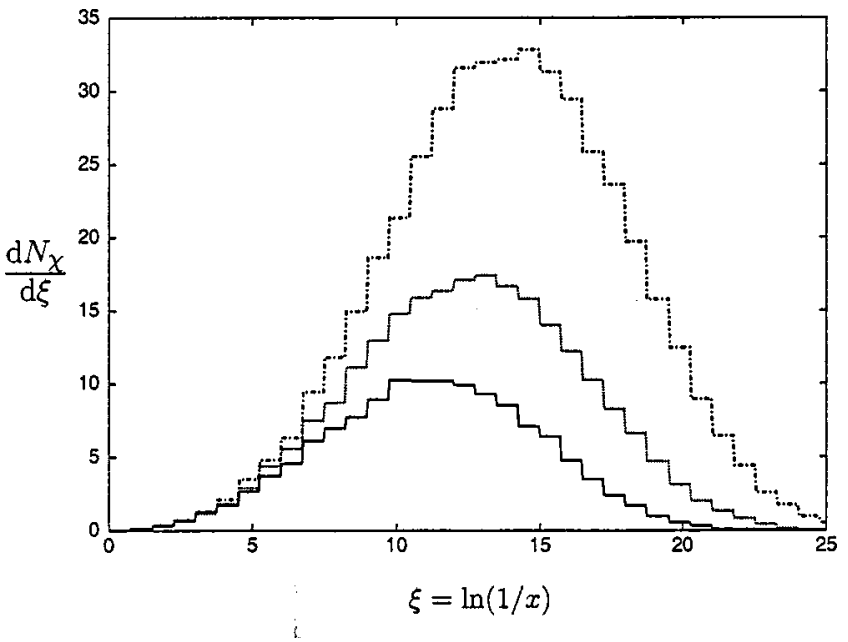

FIG. 10. Neutralino spectra from the SUSY QCD Monte Carlo simulation for $M_{X}=10^{12} \mathrm{GeV}$ (bottom), $M_{X}=10^{13} \mathrm{GeV}$ (middle) and $M_{X}=10^{14} \mathrm{GeV}$ (top), with all for $M_{\mathrm{SUSY}}=200 \mathrm{GeV}$.

stable neutralinos. They are generated in the cascade mostly when the virtuality of the spartons approaches $M_{\text {SUSY }}^{2}$. The calculated neutralino spectra are shown in Figs. 10-13 for the same parameters as the hadron spectra in Figs. 6-9. Like the hadron spectra, they have the characteristic Gaussian form, however, with a shifted position of their maxima due to their larger cutoff $M_{\text {SUSY }}$ in the shower development. The energy fraction taken away by the neutralinos is typically $10 \%$ for values of $M_{X}$ interesting for UHECR physics, with a minimum of $5 \%$ for $M_{X}=10^{6} \mathrm{GeV}$ and $M_{\text {SUSY }}=1 \mathrm{TeV}$, and a maximum of $12 \%$ for $M_{X}=10^{16} \mathrm{GeV}$ and $M_{\text {SUSY }}$ $=200 \mathrm{GeV}$.

We have only derived a common hadronization function for all hadrons and, consequently, we cannot calculate directly, e.g. pion or nucleon spectra, through Eq. (8). Since the fraction of energy $\epsilon_{i}$ going into different meson and baryon species is determined by the non-perturbative process of hadronization, these fractions as the hadronization func-

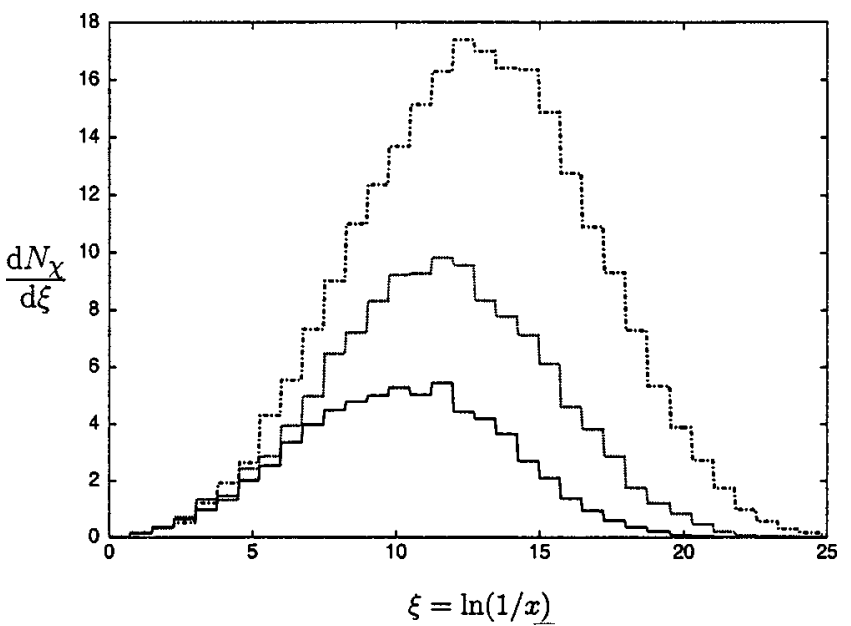

FIG. 11. Neutralino spectra from the SUSY QCD Monte Carlo simulation for $M_{X}=10^{12} \mathrm{GeV}$ (bottom), $M_{X}=10^{13} \mathrm{GeV}$ (middle) and $M_{X}=10^{14} \mathrm{GeV}$ (top), with all for $M_{\mathrm{SUSY}}=1 \mathrm{TeV}$. 


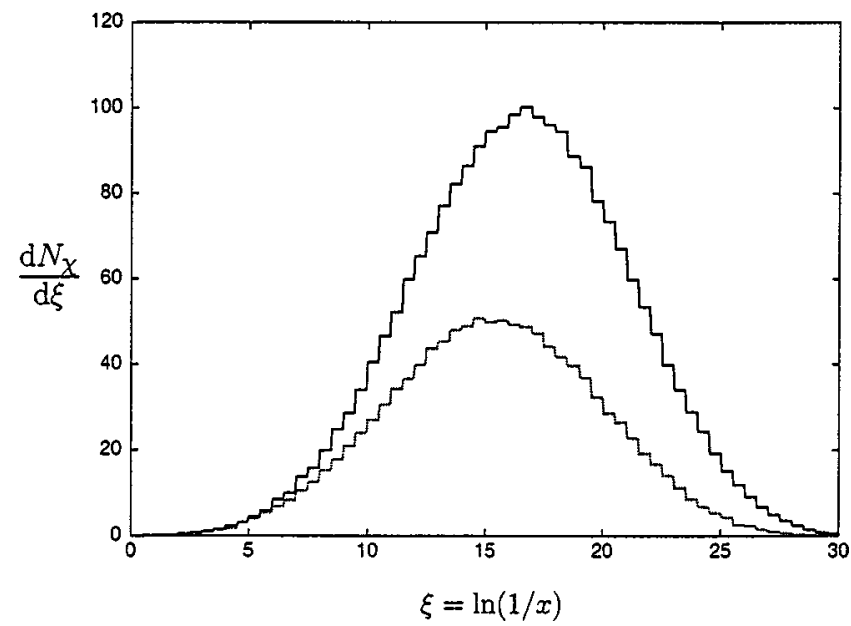

FIG. 12. Neutralino spectra from the SUSY QCD Monte Carlo simulation for $M_{\text {SUSY }}=200 \mathrm{GeV}$ (top) and $M_{\mathrm{SUSY}}=1 \mathrm{TeV}$ (bottom), both for $M_{X}=10^{16} \mathrm{GeV}$.

tions themselves do not depend on $s$. Thus, we can use the values from $Z$ decay, $\epsilon_{N} \approx 0.05$ and $\epsilon_{\pi} \approx 0.95$. Then

$$
\frac{\mathrm{d} N_{\text {nucl }}}{\mathrm{d} x}=\epsilon_{N} \frac{\mathrm{d} N_{\mathrm{had}}}{\mathrm{d} x}, \quad \frac{\mathrm{d} N_{\pi}}{\mathrm{d} x}=\epsilon_{\pi} \frac{\mathrm{d} N_{\mathrm{had}}}{\mathrm{d} x} .
$$

Using the hadron spectra obtained in the last section, it is simple to calculate analytically the spectra of secondary particles, photons and neutrinos. The normalized photon spectrum from a decay of one $X$ particle at rest is given by

$$
\frac{\mathrm{d} N_{\gamma}}{\mathrm{d} x}=\frac{2}{3} \epsilon_{\pi} \int_{x}^{1} \frac{\mathrm{d} y}{y} \frac{\mathrm{d} N_{\mathrm{had}}}{\mathrm{d} y} .
$$

The total neutrino spectrum, given by the sum from decays of pions and muons, can be presented in the following form:

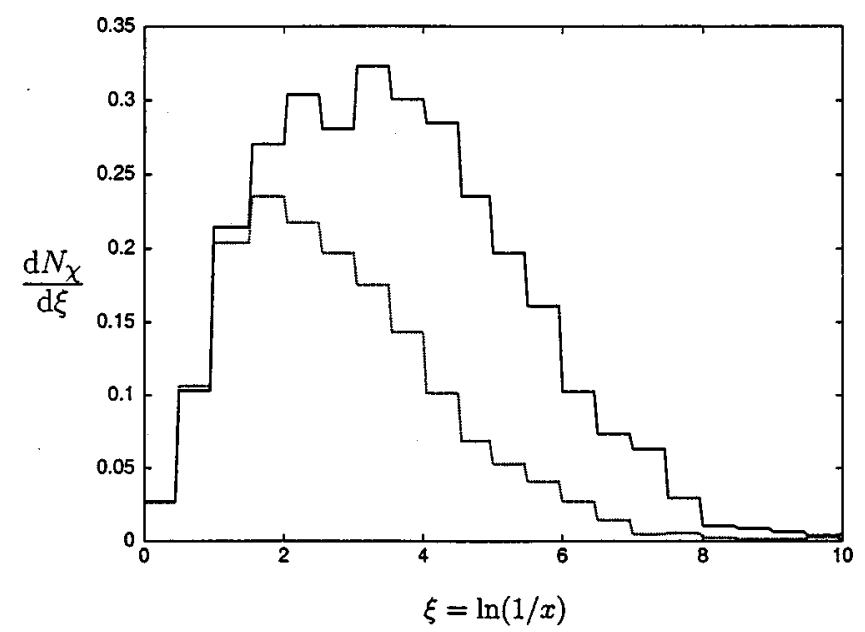

FIG. 13. Neutralino spectra from the SUSY QCD Monte Carlo simulation for $M_{\text {SUSY }}=200 \mathrm{GeV}$ (top) and $M_{\text {SUSY }}=1$ TeV (bottom), both for $M_{X}=10^{6} \mathrm{GeV}$.

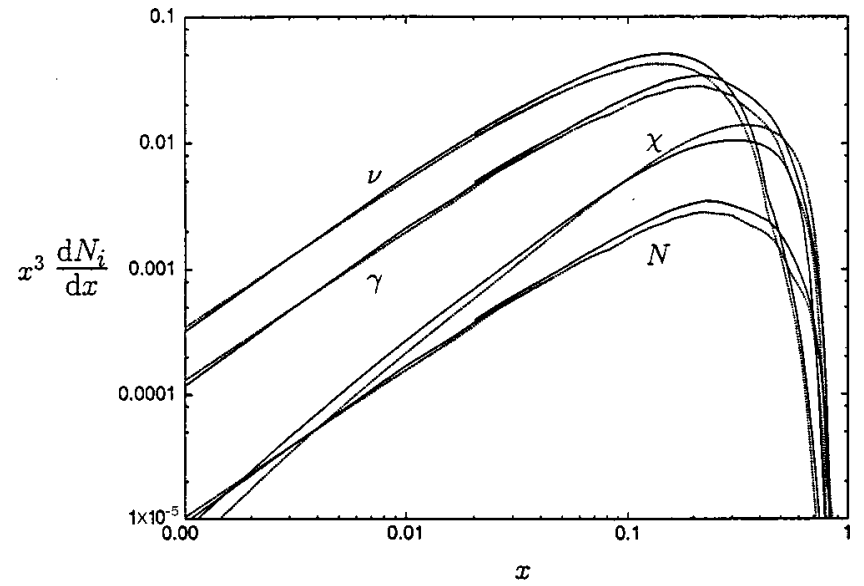

FIG. 14. Neutrino, gamma and nucleon fragmentation spectra from the SUSY QCD Monte Carlo simulations for $M_{X}=10^{12} \mathrm{GeV}$ (solid lines) and $10^{14} \mathrm{GeV}$ (dotted lines), both for $M_{\mathrm{SUSY}}=200$ $\mathrm{GeV}$.

$$
\begin{aligned}
\frac{\mathrm{d} N_{\nu}}{\mathrm{d} x}= & \frac{2}{3} \epsilon_{\pi}\left(\frac{\mathrm{d} N_{\nu_{\mu}}}{\mathrm{d} x}\left(\pi \rightarrow \mu \nu_{\mu}\right)+\frac{\mathrm{d} N_{\nu_{\mu}}}{\mathrm{d} x}\left(\mu \rightarrow \nu_{\mu} \nu_{e} e\right)\right. \\
& \left.+\frac{\mathrm{d} N_{\nu_{e}}}{\mathrm{~d} x}\left(\mu \rightarrow \nu_{\mu} \nu_{e} e\right)\right),
\end{aligned}
$$

where for pion decay

$$
\frac{d N_{\nu_{\mu}}}{\mathrm{d} x}\left(\pi \rightarrow \mu \nu_{\mu}\right)=R \int_{R x}^{1} \frac{\mathrm{d} y}{y} \frac{\mathrm{d} N_{\mathrm{had}}}{\mathrm{d} y}
$$

and for muon decay

$$
\frac{\mathrm{d} N_{\nu_{i}}}{\mathrm{~d} x}\left(\mu \rightarrow \nu_{\mu} \nu_{e} e\right)=R \int_{x}^{1} \frac{\mathrm{d} y}{y} \int_{y}^{y / r} \frac{\mathrm{d} y^{\prime}}{y^{\prime}} \frac{\mathrm{d} N_{\nu_{i}}}{\mathrm{~d} y} \frac{\mathrm{d} N_{\mathrm{had}}}{\mathrm{d} y^{\prime}},
$$

with

$$
\frac{\mathrm{d} N_{\nu_{e}}}{\mathrm{~d} y}=2-6 y^{2}+4 y^{3}, \quad \frac{\mathrm{d} N_{\nu_{\mu}}}{\mathrm{d} y}=\frac{5}{3}-3 y^{2}+\frac{4}{3} y^{3},
$$

and $r=\left(m_{\mu} / m_{\pi}\right)^{2}, R=1 /(1-r)$.

The resulting nucleon, photon and neutrino spectra $x^{3} \mathrm{~d} N_{i} / \mathrm{d} x$ are shown as functions of $x$ together with the spectra of neutralinos in Fig. 14 for $M_{\text {SUSY }}=200 \mathrm{GeV}$ and $M_{X}$ $=10^{12} \mathrm{GeV}$ and $M_{X}=10^{14} \mathrm{GeV}$, respectively. We have multiplied the spectra by $x^{3}$ in order to facilitate the comparison of our spectra with the energy spectra of observed UHECR. At $x \gtrsim 0.7$, the spectra have some uncertainties because of the unknown branching ratios of the $X$ particle into (s)partons and fluctuations due to the small number of produced particles.

\section{DISCUSSION}

In this section we compare the results of our MC simulations for large $M_{X}$ for the two cases, SUSY QCD and ordinary QCD, with other computations, and most notably, with 


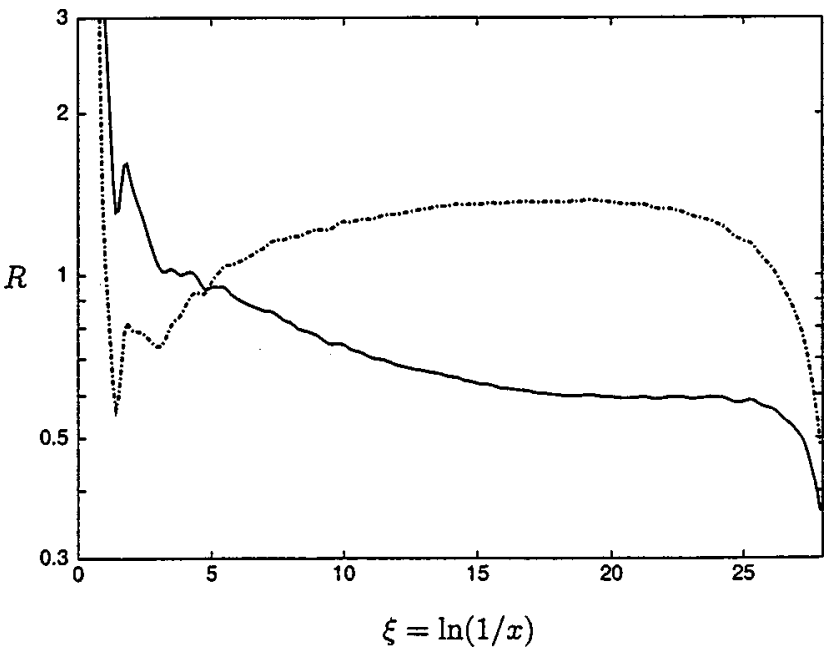

FIG. 15. Comparison of the limiting spectrum of QCD for $n_{f}$ $=3$ and $n_{f}=6$ with the ordinary QCD spectrum from the Monte Carlo simulation: $R=D_{\lim }\left(\mathrm{QCD}, n_{f}=3\right) / D_{\mathrm{MC}}$ (solid line) and $R$ $=D_{\lim }\left(\mathrm{QCD}, n_{f}=6\right) / D_{\mathrm{MC}}$ (broken line). Both are for $M_{X}=10^{12}$ $\mathrm{GeV}$.

limiting spectrum calculations. The latter case of ordinary QCD is formally a special case of our MC simulation for SUSY QCD in the limit $M_{\mathrm{SUSY}}, t_{\mathrm{SUSY}} \rightarrow \infty$, i.e. $\alpha_{s}$ is given by two-loop approximation with variable $n_{f}$, and the probability to produce a sparton is zero.

The validity of our method has been proved by the tests described in Sec. III. If no new physics beyond the three light quark flavors is introduced, the $k_{\perp}$-dependence of $\alpha_{s}$ is given by Eq. (1) with $n_{f}=3$ and the limiting spectrum with $K_{h}=1.3$ is valid for arbitrary high energies. We can calculate the hadron spectrum in our ordinary QCD MC simulations (hadronization procedure included), introducing there the same assumptions about $n_{f}$ and $\alpha_{s}$. The excellent agreement is illustrated by Fig. 5. The disagreement seen at large $x$ is natural because the limiting spectrum is not valid there.

It is instructive to compare our MC simulation for ordinary QCD with varying $n_{f}$, and the exact behavior of $\alpha_{s}\left(k_{\perp}\right)$ with the limiting spectrum with fixed number of flavors $n_{f}=3$ and $n_{f}=6$. It is clear that, in either case, $\alpha_{s}\left(k_{\perp}\right)$ from Eq. (1) does not correctly describe $\alpha_{s}$ in the whole interval of $k_{\perp}$, and the MC spectrum should be between these two solutions. Figures 15 and 16 show that this is indeed the case. The accuracy of each limiting spectrum compared with the MC spectra is better than 30-50\%.

In Ref. [30], HERWIG was used to obtain fragmentation spectra in the case of ordinary QCD. The maximal mass $M_{X}$ possible to simulate was $M_{X}=10^{11} \mathrm{GeV}$, and even for this not very large value of $M_{X}$ the computations required several months. The spectra were displayed only for large $x>0.01$, beyond the Gaussian peak. One of the conclusions of this work was that at large $x$ the proton yield is higher than the photon and neutrino yield. However, it was later realized that this result is caused by the tendency of HERWIG to overproduce protons at large $x$ (Ref. [31], but see also [32]).

Let us come over to our SUSY QCD MC simulation and compare the simulated spectra with the SUSY limiting spec-

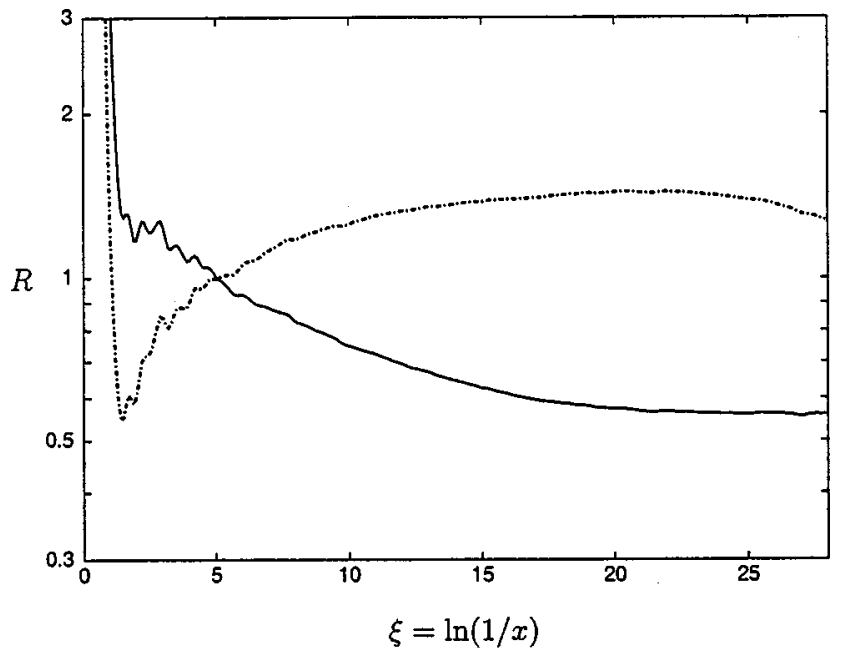

FIG. 16. Comparison of the limiting spectrum of QCD for $n_{f}$ $=3$ and $n_{f}=6$ with the ordinary QCD spectrum from the Monte Carlo simulation: $R=D_{\lim }\left(\mathrm{QCD}, n_{f}=3\right) / D_{\mathrm{MC}}$ (solid line) and $R$ $=D_{\lim }\left(\mathrm{QCD}, n_{f}=6\right) / D_{\mathrm{MC}}$ (broken line). Both are for $M_{X}=10^{14}$ $\mathrm{GeV}$.

trum [21]. The spectra disagree both in the position of the Gaussian peak and in its height. To clarify which assumptions of the SUSY QCD limiting spectrum are responsible for this disagreement, we rerun the SUSY QCD MC simulation with a set of assumptions as similar as possible to those used in the derivation of the SUSY QCD limiting spectrum. We found that the main reason for the disagreement is the universal dependence of $\alpha_{s}(t)$, taken as $\alpha_{s}^{-1}(t)$ $=\left(b_{s} / 4 \pi\right) \ln \left(t / \Lambda^{2}\right)$, with $b_{s}=3$ for SUSY, together with $\Lambda$ $=Q_{0}=250 \mathrm{MeV}$. It differs from $\alpha_{s}$ with a variable number of flavors, which is used in the SUSY QCD MC simulation, by a factor $1.4-3$ in the whole $k_{\perp}^{2}$ range, with the largest disagreement at small $k_{\perp}$. Changing the evolution of $\alpha_{s}\left(k_{\perp}\right)$, an agreement can be reached between the MC simulation and the SUSY QCD limiting spectrum: we run the SUSY QCD MC simulation including only gluons and (massless) gluinos with fixed $b_{s}=3$, and with frozen $\alpha_{s}(\tilde{t})$ for $\tilde{t}<0.9 \mathrm{GeV}^{2}$, which is a reasonable physical assumption. The comparison with the SUSY QCD limiting spectrum for partons is shown in Fig. 17. The two spectra agree quite well indeed.

An interesting alternative approach to computing the fragmentation spectra produced by decays of superheavy particles was suggested in a recent work [31]. In this method, the event generator SPYTHIA [33] was used to simulate fragmentation spectra of partons and spartons into protons, photons, and neutrinos at the scale $M_{X}=10^{4} \mathrm{GeV}$. Then the DGLAP equations were used to evolve the fragmentation functions up to the scale $10^{12}-10^{13} \mathrm{GeV}$.

It is premature to compare our results, since in [31] preliminary results are presented, but the spectra, as displayed in [31] and [32], do not agree well with ours. In particular, the Gaussian peak is broader than in our calculations, shown in Fig. 14. Comparing these spectra one should be aware of the differences in methods and assumptions. For example, we 


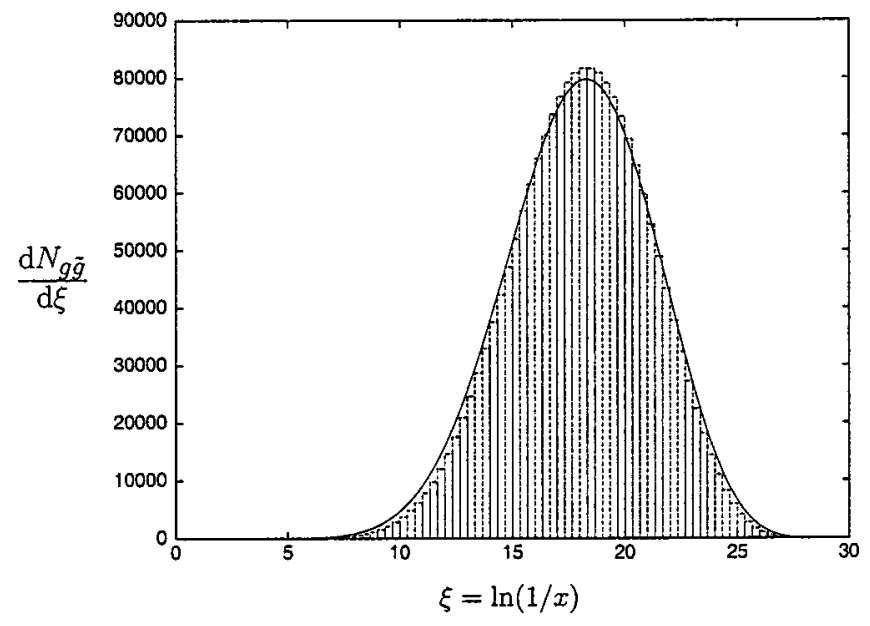

FIG. 17. Parton spectrum from the SUSY QCD Monte Carlo simulation (boxes) and the SUSY QCD limiting spectrum (solid line) for $M_{X}=10^{12} \mathrm{GeV}$. Both are for gluons and gluinos only. The coupling constant in the Monte Carlo simulation is frozen at $\tilde{t}$ $<0.9 \mathrm{GeV}^{2}$, and $b_{s}=3$ is fixed in both cases.

treat spartons as cascading particles, while in SPYTHIA spartons are taken as on-shell particles, which decay but do not cascade. On the other hand, the SPYTHIA spectrum is used only as the input, and the evolution to higher energies includes cascading. This difference will be eliminated with the $\mathrm{C}++$ version of HERWIG [23], which will be available soon.

\section{SUMMARY}

We have developed a new MC simulation for jet fragmentation in ordinary QCD and SUSY QCD, which is valid for initial energies up to the GUT scale. The simulation includes a perturbative part, operating at virtualities higher than the infrared cutoff $Q_{0}^{2}=0.625 \mathrm{GeV}$, and a hadronization part.

The perturbative part for SUSY QCD includes squarks and gluinos as cascade particles with a universal mass
$M_{\text {SUSY }}$. The evolution of $\alpha_{s}(t)$ takes into account the correct number of active flavors and spartons at a given $t$. As one can see from Figs. 8 and 9, the influence of the scale $M_{S U S Y}$ on the hadron spectrum is rather weak for the studied range $300 \leqslant M_{S U S Y} \leqslant 1000 \mathrm{GeV}$, and it remains equally weak in case the mass spectrum of SUSY particles is spread over this interval.

The hadronization scheme is model-independent and based on the well justified and tested assumption that the hadronization function $f_{i}^{h}(z)$ [see Eq. (8)], does not depend on $\sqrt{s}$. Thus the hadronization function could be calculated from LEP data. Our scheme was tested at $\sqrt{s}=58$ and 133 $\mathrm{GeV}$ against experimental data, and for very large values of $M_{X}$ by a comparison with the limiting spectrum solution for ordinary QCD. For the aim of this comparison, we calculated hadron spectra using the MC simulation for ordinary QCD in the case when the limiting spectrum is known to be correct: $n_{f}=3$ and $\alpha_{s}(t)$, given by Eq. (1). The excellent agreement between both spectra is illustrated by Fig. 5 .

The spectra of nucleons and secondary particles, photons, neutrinos, as well as neutralinos, have been calculated and presented in Fig. 14. These spectra can be used for calculations of fluxes of ultra high energy cosmic rays, produced by superheavy dark matter and topological defects.

\section{ACKNOWLEDGMENTS}

We acknowledge with gratitude the participation of $\mathrm{R}$. Sang in the initial stage of this work. We are grateful to $\mathrm{Yu}$. L. Dokshitzer, V. A. Khoze, S. S. Ostapchenko and B. R. Webber for many useful explanations and comments. M. K. would like to thank the Alexander von Humboldt-Stiftung for a Feodor-Lynen grant and the EC for a Marie-Curie grant. The work of M. K. was also partially supported by DGICYT grant PB95-1077 and by the EC under the TMR contract ERBFMRX-CT96-0090. The work by V. B. was performed within INTAS Project No. 99-01065.
[1] V.N. Gribov and L.N. Lipatov, Yad. Fiz. 15, 781 (1972) [Sov. J. Nucl. Phys. 15, 438 (1972)]; 15, 1218 (1972) [ 15, 675 (1972)]; G. Altarelli and G. Parisi, Nucl. Phys. B126, 298 (1977); Yu.L. Dokshitzer, Zh. Éksp. Teor. Fiz. 73, 1216 (1977) [Sov. Phys. JETP 46, 641 (1977)].

[2] Yu. L. Dokshitzer and S. I. Troyan, Leningrad Preprint No. LNPI-922, 1984; Ya.I. Azimov, Yu.L. Dokshitzer, V.A. Khoze, and S.I. Troyan, Z. Phys. C 27, 65 (1985); 31, 213 (1986).

[3] A. H. Mueller, Proceedings of the 1981 International Symposium on Lepton and Photon Interactions, edited by W. Pfeil and Bonn, 1981 p. 689; Yu.L. Dokshitzer, V.S. Fadin, and V.A. Khoze, Phys. Lett. 115B, 242 (1982).

[4] C.P. Fong and B.R. Webber, Phys. Lett. B 229, 289 (1989); Nucl. Phys. B355, 54 (1991).

[5] Yu. L. Dokshitzer, V. A. Khoze, A. H. Mueller, and S. I. Troyan, Basics of Perturbative QCD (Editions Frontières, Gifsur-Yvette, 1991).
[6] V.A. Khoze and W. Ochs, Int. J. Mod. Phys. A 12, 2949 (1997).

[7] S. Bethke, J. Phys. G 26, R27 (2000).

[8] V.A. Khoze, S. Lupia, and W. Ochs, Phys. Lett. B 386, 451 (1996).

[9] S. Lupia and W. Ochs, Eur. Phys. J. C 2, 307 (1998).

[10] G. Marchesini and B.R. Webber, Nucl. Phys. B238, 1 (1984).

[11] A.H. Mueller, Phys. Lett. 104B, 161 (1981); B.I. Ermolaev and V.S. Fadin, Pis'ma Zh. Éksp. Teor. Fiz. 33, 285 (1981) [JETP Lett. 33, 269 (1981)]; A. Bassetto, M. Ciafaloni, G. Marchesini, and A.H. Mueller, Nucl. Phys. B207, 189 (1982); Yu.L. Dokshitzer, V.S. Fadin, and V.A. Khoze, Phys. Lett. 115B, 242 (1982).

[12] G. Marchesini, B.R. Webber, G. Abbiendi, I.G. Knowles, M.H. Seymour, and L. Stanco, Comput. Phys. Commun. 67, 465 (1992); G. Corcella, I.G. Knowles, G. Marchesini, S. Moretti, K. Odagiri, P. Richardson, M.H. Seymour, and B.R. Webber, hep-ph/9912396. 
[13] T. Sjöstrand, Comput. Phys. Commun. 82, 74 (1994).

[14] L. Lönnblad, Comput. Phys. Commun. 71, 15 (1992).

[15] H. Baer, F.E. Paige, S.D. Protopopescu, and X. Tata, hep-ph/0001086.

[16] Ya.I. Azimov, Yu.L. Dokshitzer, V.A. Khoze, and S.I. Troyan, Phys. Lett. 165B, 147 (1985); see second reference in [2].

[17] D. Amati and G. Veneziano, Phys. Lett. 83B, 87 (1979); A. Bassetto, M. Ciafaloni, and G. Marchesini, ibid. 83B, 207 (1979); G. Marchesini, L. Trentadue, and G. Veneziano, Nucl. Phys. B181, 335 (1980).

[18] B. Anderson, G. Gustafson, G. Ingelman, and T. Sjöstrand, Phys. Rep. 97, 31 (1983).

[19] B.R. Webber, Nucl. Phys. B238, 492 (1984).

[20] V. Berezinsky, Nucl. Phys. B (Proc. Suppl.) 87, 387 (2000); P. Bhattacharjee and G. Sigl, Phys. Rep. 327, 109 (2000).

[21] V. Berezinsky and M. Kachelrieß, Phys. Lett. B 434, 61 (1998).

[22] R. K. Ellis, W. J. Stirling, and B. R. Webber, QCD and Collider Physics (Cambridge University Press, Cambridge, England, 1996).
[23] B. R. Webber (private communication).

[24] T. Sjöstrand, PYTHIA 5.7 and JETSET 7.4 Physics and Manual, Preprint Nos. LU TP 95-20 and CERN-TH 7112/93.

[25] S.K. Jones and C.H. Llewellyn-Smith, Nucl. Phys. B217, 145 (1983), and references therein.

[26] U. Amaldi, W. de Boer, and H. Fürstenau, Phys. Lett. B 260, 447 (1991); J. Ellis, S. Kelly, and D.V. Nanopoulos, ibid. 260, 131 (1991).

[27] P. Langacker and N. Polonsky, Phys. Rev. D 47, 4028 (1993).

[28] I. J. D. Craig and J. C. Brown, Inverse Problems in Astronomy (Adam Hilger, London, 1986).

[29] TOPAZ Collaboration, R. Itoh et al., Phys. Lett. B 345, 335 (1995); DELPHI Collaboration, P. Abreu et al., Z. Phys. C 73, 11 (1996); OPAL Collaboration, G. Alexander et al., ibid. 72, 191 (1996).

[30] M. Birkel and S. Sarkar, Astropart. Phys. 9, 297 (1998).

[31] N. A. Rubin, Master's thesis, University of Cambridge, 1999.

[32] S. Sarkar, hep-ph/0005256.

[33] S. Mrenna, Comput. Phys. Commun. 101, 232 (1997). 